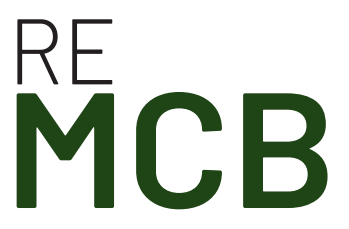

Revista Ecuatoriana de Medicina y Ciencias Biológicas

Volumen 42. No. 1, Mayo 2021

\section{El losartán disminuye la expresión del receptor para compuestos de glicación avanzada en el tejido cardiaco de ratas diabéticas}

\author{
Losartan Decreases Receptor Expression for Advanced Glycation Compounds \\ in Heart Tissue of Diabetic Rats
}

Nelson Muñóz', Jesús Mosquera², Adriana Pedreáñez ${ }^{3 *}$
'Universidad Nacional del Chimborazo. Facultad de Ciencias de la Salud. Riobamba. Ecuador. ¿Universidad del Zulia, Facultad de Medicina, Instituto de Investigaciones Clínicas "Dr. Américo Negrette". Maracaibo, Venezuela.

${ }^{3}$ Universidad del Zulia, Facultad de Medicina, Escuela de Bioanálisis. Maracaibo, Venezuela.

*Autor de Correspondencia: apedreanez@gmail.com, ORCID: https//orcid.org/0000-0002-39370469

Recibido 7-12-2020

Aceptado 6-04-2021

DOI: 10.26807/remcb.v42i1.883

e-ISSN 2477-9148

(c) 2021. Este es un artículo publicado en acceso abierto bajo una licencia CC BY 4.0

Como citar este artículo: Muñoz N, Mosquera J, Pedreáñez A. 2021. El losartán disminuye la expresión del receptor para compuestos de glicosilación avanzada en el tejido cardiaco de ratas diabéticas. Revista Ecuatoriana de Medicina y Ciencias Biológicas 42(1): 27-36. doi: 10.26807/remcb. v42i1.883
Resumen.- El receptor para productos finales de glicación avanzada (RAGE) puede interactuar con diversos ligandos e inducir procesos inflamatorios. El objetivo de esta investigación fue determinar el efecto del enalapril y losartán sobre la expresión de RAGE en el tejido cardíaco en un modelo experimental de diabetes. Se utilizaron ratas Sprague-Dawley machos, con un peso de 150 - $200 \mathrm{~g}$. La diabetes se indujo en 30 ratas mediante la administración intravenosa de una sola dosis de $55 \mathrm{mg} / \mathrm{Kg}$ de peso corporal de estreptozotocina (ETZ). Se estudiaron los siguientes grupos: ratas control, diabéticas, diabéticas tratadas con losartán y diabéticas tratadas con enalapril. La expresión de RAGE en miocardio se determinó por inmunofluorescencia indirecta. Se observó un aumento significativo en la expresión de RAGE en los animales diabéticos versus los controles $(p<0.01)$, hubo una disminución en la expresión de RAGE miocárdico, sólo en los animales tratados con losartán versus los diabéticos sin tratamiento $(p<0.05)$. En conclusión, en el modelo experimental de diabetes inducida por ETZ, existe un incremento en la expresión miocárdica de RAGE, la cual puede revertirse mediante el tratamiento con losartán lo cual indica la participación del sistema renina angiotensina en los mecanismos relacionados con el daño cardiaco durante la diabetes.

Palabras clave: diabetes, enalapril, losartan, miocardio, RAGE

Abstract.- The receptor for advanced glycation end products (RAGE) can interact with various ligands and induce inflammatory processes. The aim of this research was to determine the effect of enalapril and losartan on the expression of RAGE in cardiac tissue in an experimental model of diabetes. Male Sprague-Dawley rats weighing 150 - $200 \mathrm{~g}$ were used. Diabetes was induced in 30 rats by intravenous administration of a single dose of $55 \mathrm{mg} / \mathrm{kg}$ body weight of streptozotocin (STZ). The following groups were studied: control, diabetic, losartan-treated and enalapril-treated diabetic rats. The RAGE expression in myocardium was determined by indirect immunofluorescence. A significant increase in the RAGE expression was observed in diabetic animals versus controls $(p<0.01)$, there was a decrease in myocardial RAGE expression, only in animals treated with losartan versus untreated diabetics $(p<0.05)$. In conclusion, in the experimental model of STZ-induced diabetes, there is an increase in myocardial RAGE expression, which can be reversed by treatment with losartan indicating the involvement of the angiotensin renin system in the mechanisms related to heart damage during diabetes.

Keywords: diabetes, enalapril, losartan, myocardial, RAGE 


\section{Introduccion}

Existe un alto índice de morbilidad y mortalidad por enfermedad cardíaca en los pacientes con diabetes (Hass y MacDonnell 2018; Varma et al. 2018; Alegria et al. 2007). Sin embargo los mecanismos involucrados en la enfermedad cardiovascular en estos pacientes permanecen bajo estudio. La hiperglicemia crónica asociada a la diabetes da lugar a la glicación no enzimática de proteínas, cuando los azúcares reductores como la glucosa reaccionan con los grupos amino libres de las proteínas, dando origen a la formación de productos finales de glicación avanzada (AGEs) por sus siglas en inglés (advanced glycation end products) (Stefano et al. 2016; Kehm et al. 2019).

Cuando los AGEs se unen a su receptor específico RAGE (receptor for advanced glycation end products) se desencadenan una serie de respuestas intracelulares que traen como consecuencia la producción de mediadores proinflamatorios los cuales han sido implicados en la patogénesis de las complicaciones durante la diabetes. (Leung et al. 2016). Varios mecanismos moleculares asociados a la diabetes promueven la inflamación del miocardio. La vía de señalización que involucra al factor de transcripción NF-kB y el sistema renina - angiotensina (SRA) están fuertemente activados en el corazón diabético y por lo tanto promueven la inflamación en dicho tejido. (Teissier y Boulanger 2019; Kass 2003).

Se ha descrito que los fármacos que bloquean el SRA, bien sea inhibidores de la enzima convertidora de angiotensina (ECA) como el enalapril o antagonistas de los receptores AT1 como el losartan, poseen un efecto beneficioso sobre las alteraciones cardiacas durante la diabetes. (Scheen 2004; El Desoky 2011). Los datos sugieren que tanto la angiotensina II como la activación de RAGE están relacionadas con la inflamación y el desarrollo de las complicaciones cardiacas, sin embargo las interacciones entre ambas moléculas durante la enfermedad permanece desconocida. Es por ello que el objetivo de esta investigación fue evaluar el efecto del enalapril y el losartán sobre la expresión de RAGE en el tejido cardiaco de ratas con diabetes inducida experimentalmente.

\section{Materiales y Métodos}

Inducción de diabetes experimental con estreptozotocina .- Para el estudio se utilizaron ratas Sprague-Dawley machos con peso aproximado de 150 - 200 g, procedentes del bioterio del Instituto Venezolano de Investigaciones Científicas (IVIC), Altos de Pipe del Estado Miranda. El modelo experimental de diabetes se indujo en 30 ratas mediante la administración intravenosa (i.v) de una sola dosis de $(55 \mathrm{mg} / \mathrm{Kg}$ de peso corporal de estreptozotocina (ETZ); Sigma-Aldrich, St. Louis, MO, USA) diluida en buffer citrato $10 \mathrm{mM}$, $\mathrm{pH} 4,5$. Los animales controles $(n=10)$ recibieron buffer citrato $10 \mathrm{mM}, \mathrm{pH} 4,5$ i.v.

Durante tres días luego de la inducción de la diabetes, se les suministró a los animales glucosa al 5\% como sustituto del agua. La aparición de la hiperglicemia se determinó mediante la técnica de la glucosa oxidasa/peroxidasa utilizando un monitor Prestige IQ Smart System, en sangre extraída de la cola del animal una semana luego de inducir la diabetes.

Protocolo experimental. - Los animales que desarrollaron hiperglicemia después de una semana y se mantuvieron con valores de glucosa sanguínea mayores a 200 mg/dl durante 4 semanas, se dividieron a su vez en tres grupos dos de los cuales recibieron tratamiento durante 4 semanas adicionales con inhibidores del sistema renina - angiotensina.

Grupo ETZ + Vehículo: recibió vía oral 500 ul de agua destilada. Grupo ETZ + Losartan: recibió vía oral 15 mg/kg/día de losartan. (losartan sodico; Merck \& Co, Kenilworth, New Jersey, United States). Grupo ETZ + Enalapril: recibió vía oral 18 mg/kg/día de enalapril. (Merck Sharp \& Dohme, Chalfont, Pennsylvania, United States) (Muñoz et al. 2011).

El losartán es un antagonista de los receptores AT1 de la Angiotensina II (Smith 2008); mientras que el enalapril es un inhibidor de la enzima convertidora de angiotensina I (Vargas et al. 2012). Las drogas y el agua destilada se administraron diariamente por gavaje gástrico desde la cuarta semana de diabetes hasta la octava semana. Todos los animales fueron sacrificados a la octava semana. El peso de los animales se monitoreó antes de inyectar el agente diabetogénico (ETZ) 
o el vehículo, antes de comenzar el tratamiento con losartan o enalapril, y al momento de los sacrificios.

Una vez finalizadas las 4 semanas de tratamiento, los animales se anestesiaron utilizando una inyección intraperitoneal de $100 \mathrm{mg} / \mathrm{Kg}$ de tiobarbitúrico y se les realizó una laparotomía. Se hizo una punción a la aorta abdominal para la toma de muestra de sangre; seguidamente se abrió la cavidad torácica y se perfundió el corazón con solución salina fisiológica 0.9 \% a través del ventrículo izquierdo. Posteriormente se procedió a la toma de biopsias de corazón las cuales fueron embebidas en Tissue Tek (Miles Inc. Diagnostic Division, Kankakee, USA) y congeladas utilizando una mezcla de hielo seco y acetona y almacenadas a $-70^{\circ} \mathrm{C}$ hasta su utilización para los estudios de inmunofluorescencia. Todos los experimentos se realizaron siguiendo los lineamientos del comité de bioética y bioseguridad del FONACYT y el Comité de Bioética de la Facultad de Medicina de la Universidad del Zulia (Maracaibo, Venezuela).

Estudios de inmunofluorescencia para la determinación de la expresión de RAGE.- Se realizaron cortes de $4 \mu \mathrm{m}$ de las biopsias obtenidas de tejido cardiaco, se fijaron con acetona y se determinó mediante la técnica de inmunofluorescencia indirecta la expresión de RAGE (Rabbit anti-rat RAGE. ab3611; Abcam, Cambridge Biomedical Campus, Cambridge, UK).

Las secciones de tejido luego de ser fijadas, se incubaron con el anticuerpo primario correspondiente, para posteriormente ser incubadas con un anticuerpo secundario hecho en cabra conjugado con rodamina y dirigido contra lgG de conejo (goat anti-rabbit lgG rhodamine conjugated. Sigma Aldrich, St. Louis, MO, USA). Finalmente a las secciones de tejido cardíaco se les colocó una solución de p-fenilendiamina (PPD) en PBS-glicerol. La evaluación de la fluorescencia se realizaró con un microscopio dotado de un sistema de epifluorescencia (Axioscop, Zeiss, Germany). Los resultados se presentaron como células positivas por mm2 de tejido de 20 campos microscópicos seleccionados aleatoriamente.

Análisis estadístico .- Los resultados se expresan como media \pm desviación estándar. Para estudiar las diferencias entre los grupos controles y los experimentales se utilizó el análisis de varianza (ANOVA) de una vía con post-prueba de Bonferroni's para establecer comparaciones intergrupales. Se consideró como estadísticamente significativa todo valor de $p<0,05$.

\section{Resultados}

La tabla 1, muestra los pesos y la concentración de glicemia en los diferentes grupos estudiados. La inyección de ETZ produjo animales diabéticos con altos niveles de glucosa en la sangre, acompañados de una disminución de peso en comparación con los controles ${ }^{*} \mathrm{p}<0.001$ vs control). Los animales diabéticos tratados con enalapril mostraron valores similares de glucosa en la sangre a los observados en ratas diabéticas no tratadas. Por otra parte, los animales que recibieron losartan, mostraron niveles más altos de glicemia con respecto al grupo tratado con enalapril. Sin embargo, no se observó una diferencia estadísticamente significativa. El tratamiento farmacológico no tuvo efectos sobre el peso de los animales diabéticos.

El análisis inmunohistoquímico mostró un aumento en el número de células positivas para RAGE en el tejido miocárdico de los animales diabéticos (Figuras 1 y 2). Sólo los animales tratados con losartán mostraron una disminución en el número de células que expresaban RAGE a nivel de miocardio (Figura 1).

Tabla 1. Pesos y glicemias de los diferentes grupos estudiados a las 8

\begin{tabular}{ccc}
\hline Grupos & Peso $(\mathbf{g})$ & Glicemia $(\mathbf{m g} / \mathbf{d L})$ \\
\hline Controles & $364.55 \pm 26.73$ & $116.30 \pm 16.91$ \\
ETZ & $262.83 \pm 40.46^{*}$ & $430.83 \pm 103.81^{*}$ \\
ETZ-Losartan & $235.67 \pm 46.19^{*}$ & $>600^{*}$ \\
ETZ-Enalapril & $236.68 \pm 29.17^{*}$ & $523.50 \pm 40.30^{*}$ \\
\hline
\end{tabular}

${ }^{*} \mathrm{p}<0.001$ vs Control 
$\square$ control

Diabetes + vehiculo

Diabetes + losartan

Diabetes + enalapril

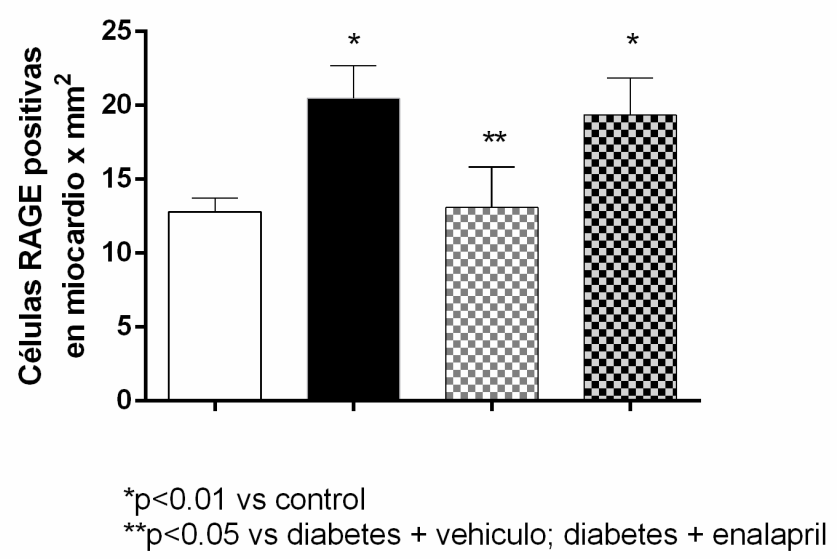

Figura 1. Expresión de RAGE en el tejido miocárdico en los diferentes grupos estudiados. Se observó un incremento en la expresión de RAGE en las ratas diabéticas el cual disminuyó con el tratamiento con losartán.
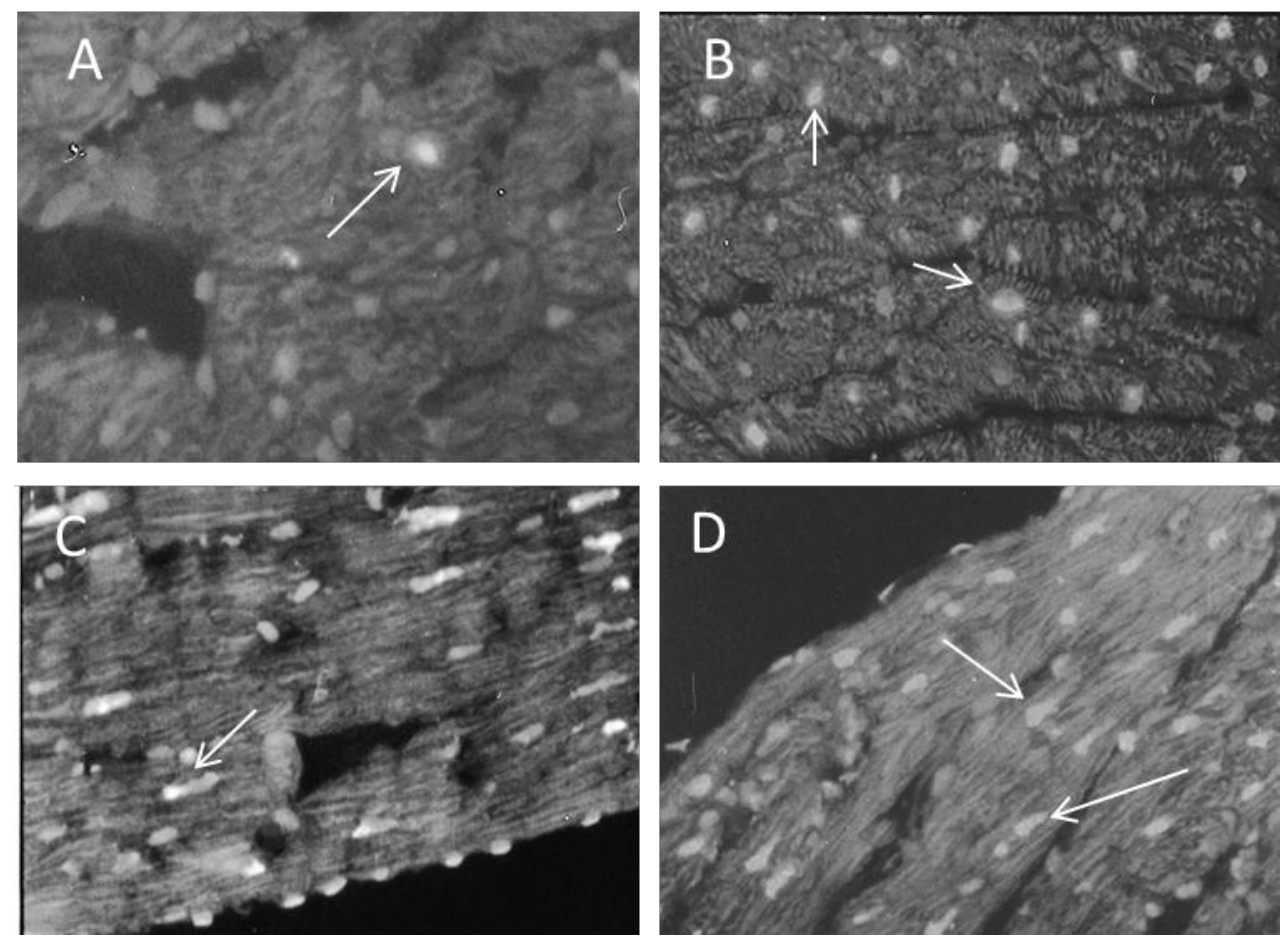

Figura 2. Expresión de RAGE en miocardio de ratas controles y ratas diabéticas. (A) Expresión de células RAGE + en el tejido miocárdico de ratas control; (B) expresión de RAGE en miocardio de ratas diabéticas no tratadas que recibieron sólo vehículo; (C) Expresión disminuida de RAGE en el tejido cardiaco de ratas diabéticas tratadas con losartan; (D) ratas diabéticas tratadas con enalapril. Las flechas señalan células RAGE positivas. Magnificación original 600X. 


\section{Discusión}

Las complicaciones cardiovasculares son la principal causa de mortalidad y morbilidad en los pacientes diabéticos. Los cambios en la estructura y función del miocardio que se desarrollan como consecuencia de la diabetes se han denominado cardiomiopatía diabética (Haas y McDonnell 2018). A partir del análisis de varios modelos animales de diabetes tipo 1 y tipo 2, se han propuesto numerosos mecanismos moleculares que pueden contribuir al desarrollo de esta alteracion (Mozaffarian et al. 2015; McClelland et al. 2015; Bugger y Abel 2014). En la actualidad hay suficiente evidencia que sugiere que los pacientes diabéticos están sometidos a un gran número de perturbaciones celulares y moleculares que causan anormalidades estructurales y funcionales en el miocardio y en la vasculatura, conduciendo a inflamación, fibrosis y posterior desarrollo de cardiomiopatía diabética (Bugger y Abel 2014).

Se ha demostrado que la hiperglicemia induce la formación de AGEs y la expresión de su receptor RAGE, lo cual trae como consecuencia la activación de procesos inflamatorios. En este sentido, varios trabajos de investigación han reportado altos niveles plasmáticos de AGEs tanto en pacientes con diabetes como en modelos animales de diabetes experimental (Stefano et al. 2016; Kehm et al. 2019). La unión de RAGE a los productos de glicación avanzada es un factor determinante que trae como consecuencia la activación de mecanismos inmunitarios. En general estos mecanismos convergen en la activación del factor de transcripción NF-kB el cual promueve la síntesis de citocinas, quimiocinas y moléculas de adhesión lo que contribuye al daño cardiovascular y puede resultar en inflamación crónica. En este sentido, la activación de RAGE y la posterior translocación del factor NF-kB al núcleo se ha implicado en diferentes patologías humanas, incluyendo diabetes, Alzheimer, artritis, aterosclerosis y enfermedades relacionadas con el envejecimiento (Leung et al. 2016; Teissier y Boulanger 2019) (Figura3).

La morbilidad y mortalidad debido a infarto al miocardio es alta en pacientes con diabetes (Hass y MacDonnell 2018; Varma et al. 2018; Alegria et al. 2007). Existen evidencias de que los AGEs tienen un papel en la patogénesis de las complicaciones cardíacas en pacientes con diabetes involucrando las interacciones AGE/RAGE (Kass 2003). Los AGEs son proteínas predominantemente de larga duración que se glucosilan después de la exposición prolongada a azúcares, lo que altera sus propiedades funcionales. El aumento de la formación de AGEs secundario a la hiperglicemia puede alterar las proteínas estructurales y conducir a un aumento de la rigidez del miocardio (Goldin et al. 2006). En este sentido, se ha reportado la acumulación cardiaca de AGEs en ratas con diabetes inducida por ETZ, mostrando que las alteraciones cardiovasculares durante la diabetes podrían ser iniciadas por la acumulación de AGEs y su posterior unión a RAGE con su consecuente activación (Jing et al. 2010).

En nuestro estudio, hemos identificado dos elementos claves que podrían tener un efecto sobre el daño al miocardio durante la diabetes: la hiperglicemia y el incremento en la expresión de RAGE en células cardíacas.

La relación entre la angiotensina II y los eventos cardiovasculares descritos en el modelo de diabetes experimental inducida con ETZ es desconocida. La Ang II es el principal efector biológico del SRA y se ha reconocido desde hace muchos años como un agente vasoconstrictor tanto local como sistémico, cuyas acciones pueden modificar la reabsorción de agua y sodio en los segmentos tubulares distales de la nefrona (no directamente, sino a través de la aldosterona). Además la angiotensina II es una potente molécula proinflamatoria que puede actuar sobre diferentes tejidos, incluyendo el tejido cardíaco (Muñoz et al. 2011; Wang et al. 2016; Miller y Arnold 2019). Esta acción de la angiotensina II sobre diversos tejidos es mediada a través de su unión a receptores AT1 y AT2 (Miller y Arnold 2019; Zhou et al. 2007).

La activación del SRA miocárdico promueve procesos de remodelación en el corazón. En tal sentido, se ha descrito que la acción antagonista de la aldosterona miocárdica tiene efectos beneficiosos sobre la hipertrofia de los miocitos cardíacos y la fibrosis miocárdica (Orea-Tejeda et al. 2007). Varios estudios han demostrado un aumento del contenido de tejido conectivo en corazones de ratones con diabetes por ETZ, que puede atenuarse mediante el tratamiento con el antagonista de la aldosterona (espironolactona) (Westermann et al. 2007). Por otra parte, 


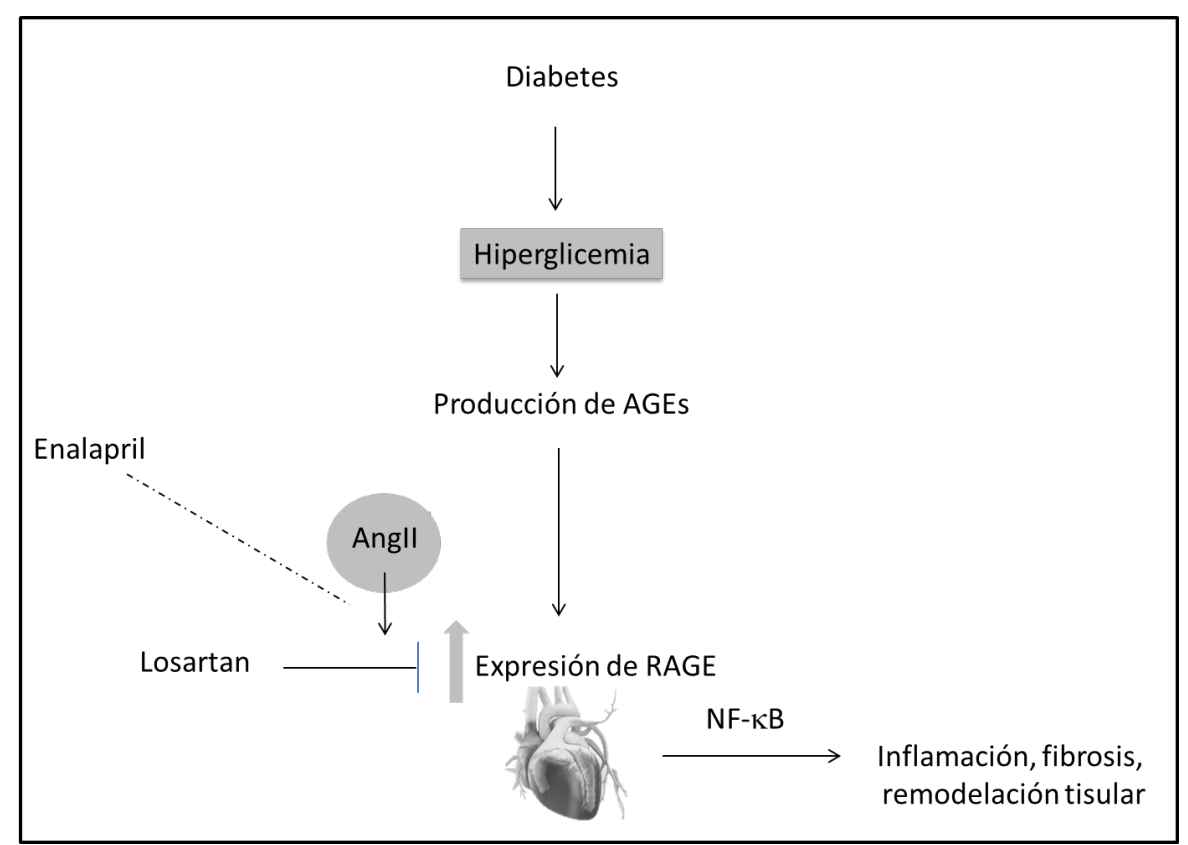

Figura 3. La hiperglicemia frecuente durante la diabetes, estimula la producción de AGEs e incrementa la expresión de RAGE en el tejido cardíaco. La activación del Eje AGEs/RAGE promueve la activación de NF-kB, lo cual trae como consecuencia la activación de mecanismos inflamatorios. La expresión de RAGE puede revertirse con el uso de losartán un antagonista de los receptores AT1 de la angiotensina II.

se ha demostrado que la densidad y síntesis del receptor de angiotensina II cardiaca aumenta en el tejido cardíaco en modelos animales de diabetes inducida por ETZ, y el aumento del estrés oxidativo y la apoptosis también pueden inhibirse, al menos parcialmente, mediante el tratamiento con bloqueadores de los receptores de angiotensina o inhibidores de la ECA (Singh et al. 2008). Adicionalmente, se ha descrito que la activación de RAGE por sus ligandos (AGEs) puede ser un efector potencial en la patología cardíaca en los pacientes con diabetes en donde están involucradas interacciones AGEs/ RAGE (Pickering et al. 2019).

En el presente estudio se observó un incremento en la expresión miocárdica de RAGE en los animales con diabetes inducida por estreptozotocina, sugiriendo un desencadenante potencial para eventos inflamatorios en el corazón. La expresión de RAGE estuvo disminuida en los animales diabéticos tratados con losartán, lo cual sugiere el papel de la angiotensina II en este proceso. Recientemente se ha reportado que la activación del receptor AT1 por la angiotensina II induce transactivación de RAGE y translocación de NF-NK-kB con la posterior expresión de genes proinflamatorios (Lim et al. 2018).

De acuerdo a este mecanismo, nuestros resultados sugieren que el losartán es capaz de bloquear el receptor AT1, de tal manera que se bloquee el efecto de la angiotensina II, lo que se traduce en una disminución en la expresión de RAGE (Muñoz et al. 2011; Goldin et al. 2006). Además se ha reportado que la hiperglicemia fue capaz de activar el sistema renina angiotensina en un modelo de diabetes mellitus inducido por ETZ (Chen et al. 2018). E incrementó la expresión de angiotensina II en vasos sanguíneos cerebrales (Goldin et al. 2006). Sugiriendo una vía de activación, angiotensina II / RAGE en este modelo experimental.

En esta investigación, se observó un efecto diferencial del enalapril y el losartán sobre la expresión de RAGE. El enalapril no fue capaz de reducir la expresión de RAGE como si lo hizo el losartán. Estos fármacos tienen un mecanismos de acción diferente. El enalapril inhibe la acción de la ECA, mientras que el losartán bloquea el efecto de la Ang II porque antagoniza sobre su 
receptor AT1 (Henriksen y Jacob 2003; Hunyady y Catt 2006), el cual media la mayoría de los efectos fisiopatológicos de la angiotensina II (vasoconstricción, estrés oxidativo, inflamación). La enzima convertidora de angiotensina cataliza la conversión de angiotensina I a angiotensina II, sin embargo se han descrito vías adicionales e independientes para la producción de angiotensina II que involucran la participación de proteasas de serina, siendo la enzima quimasa la más activa. La quimasa puede catalizar entre el 80-90\% de la conversión de la angiotensina I hacia angiotensina II en el tejido cardíaco (Kinoshita et al. 1991; Urata et al. 1991; Urata et al. 1993). Debido a esto, los resultados observados en los animales diabéticos tratados con enalapril no son inesperados.

Aunque el papel del SRA a nivel cardíaco en el modelo de diabetes inducida por ETZ no ha sido estudiado ampliamente, los antecedentes estudiados sugieren que el SRA también se activa en el corazón y está involucrado en los eventos inflamatorios. Los experimentos en el presente estudio se realizaron usando estrategias dirigidas a bloquear el efecto de Ang II, como la inhibición de la ECA y el bloqueo del receptor AT1, condiciones que han sido muy útiles para determinar los efectos de Ang II en varias pruebas clínicas y modelos experimentales de diabetes, incluyendo el modelo ETZ (Ilatovskaya et al. 2015; Chen et al. 2018; Qien at al. 2018; Ogata et al. 2016; Lozano-Maneiro y Puente-Garcia 2015). Ademas varios estudios, han demostrado un incremento significativo en la concentración de Ang II en modelos experimentales de diabetes inducida con ETZ (Fernandez-Fernandez et al. 2014; Schievink et al. 2016; Roscioni et al. 2014).

\section{Conclusión}

En el modelo experimental de diabetes inducida por ETZ, existe un incremento en la expresión miocárdica de RAGE, la cual puede revertirse mediante el tratamiento con losartán, lo cual indica la participación del sistema renina angiotensina en los acontecimientos moleculares relacionados con el daño cardiaco durante la diabetes.

\section{Agradecimientos}

Este estudio fue apoyado por el Instituto de Investigaciones Clínicas "Dr. Américo Negrette", Facultad de Medicina, Universidad del Zulia, Venezuela. No se recibió financiamiento adicional comercial, público, o de sectores sin fines de lucro.

\section{Abreviaturas}

ETZ: estreptozotocina,

RAGE: receptor para productos finales de glicación avanzada

SRA: Sistema Renina Angiotensina

ECA: Enzima convertidora de angiotensina I

Ang II: Angiotensina II

NF-kB: Factor nuclear de transcripción k-B

AGEs: Productos finales de glicación avanzada

\section{Conflicto de intereses}

Los autores declaran no poseer conflicto de intereses

\section{Contribución de los autores}

Nelson Muñoz: Adquisición/Colección de datos, Análisis e interpretación de datos, redacción. Jesús Mosquera: Concepción y diseño del estudio, Análisis e interpretación de datos y revisión del manuscrito

Adriana Pedreáñez: Revisión y Redacción de la versión final del manuscrito.

\section{Referencias bibliográficas}

Alegria JR, Miller TD, Gibbons RJ, Yi QL, Yusuf S, \& Collaborative Organization of RheothRx Evaluation (CORE) Trial Investigators. 2007. Infarct size, ejection fraction, and mortality in diabetic patients with acute myocardial infarction treated with thrombolytic therapy. Am heart J. 154(4): 743-750. 
Bugger H, Abel ED. 2014. Molecular mechanisms of diabetic cardiomyopathy. Diabetologia. 57(4): 660-671.

Chen C, Li L, Qin H, Huang Z, Xian J, Cai J, Qin Y, Zhang J, Liang X. 2018. Effects of Irbesartan Pretreatment on Pancreatic $\beta$-Cell Apoptosis in STZ-Induced Acute Prediabetic Mice. Oxid Med Cell Longev. 2018: 8616194.

Chen CM, Juan SH, Chou HC. 2018. Hyperglycemia activates the renin-angiotensin system and induces epithelial-mesenchymal transition in streptozotocin-induced diabetic kidneys. Journal of the renin-angiotensin-aldosterone system : J Renin Angiotensin Aldosterone Syst. 19(3): 1470320318803009.

El Desoky ES. 2011. Drug therapy of heart failure: an immunologic view. Am JTher. 18(5): 416-425.

Fernandez-Fernandez B, Ortiz A, Gomez-Guerrero C, Egido J. 2014. Therapeutic approaches to diabetic nephropathy--beyond the RAS. Nat Rev Nephro. 10(6): 325-346.

Goldin A, Beckman JA, Schmidt AM, Creager MA. 2006. Advanced glycation end products: sparking the development of diabetic vascular injury. Circulation. 114(6): 597-605.

Haas AV \& McDonnell ME. 2018. Pathogenesis of Cardiovascular Disease in Diabetes. Endocrinol Metab Clin North Am. 47(1): 51-63.

Henriksen EJ, Jacob S. 2003. Modulation of metabolic control by angiotensin converting enzyme (ACE) inhibition. J Cell Physiol. 196(1): 171-179.

Hunyady L, Catt KJ. 2006. Pleiotropic AT1 receptor signaling pathways mediating physiological and pathogenic actions of angiotensin II. Mol Endocrinol. 20(5): 953-970.

Ilatovskaya DV, Levchenko V, Lowing A, Shuyskiy LS, Palygin O, Staruschenko A. 2015. Podocyte injury in diabetic nephropathy: implications of angiotensin II-dependent activation of TRPC channels. Sci Rep. 5, 17637.

Jing YH, Chen KH, Yang, SH, Kuo, PC, Chen, JK. 2010. Resveratrol ameliorates vasculopathy in STZinduced diabetic rats: role of AGE-RAGE signalling. Diabetes Metab Res Re. 26(3): 212-222.

Kass DA. 2003. Getting better without AGE: new insights into the diabetic heart. Circ Res. 92(7): 704-706.

Kehm R, Rückriemen J, Weber D, Deubel S, Grune T, Höhn A. 2019. Endogenous advanced glycation end products in pancreatic islets after short-term carbohydrate intervention in obese, diabetes-prone mice. Nutr diabetes. 9(1): 9.

Kinoshita A, Urata H, Bumpus FM, Husain A. 1991. Multiple determinants for the high substrate specificity of an angiotensin II-forming chymase from the human heart. J Biol Chem. 266(29): 19192-19197.

Leung SS, Forbes JM, Borg DJ. 2016. Receptor for Advanced Glycation End Products (RAGE) in Type 1 Diabetes Pathogenesis. Curr Diab Rep. 16(10): 100.

Lim S, Lee ME, Jeong J, Lee J, Cho S, Seo M, Park S. 2018. sRAGE attenuates angiotensin I-induced cardiomyocyte hypertrophy by inhibiting RAGE-NFKB-NLRP3 activation. Inflamm Res. 67(8): 691-701.

Lozano-Maneiro L, Puente-García A. 2015. Renin-Angiotensin-Aldosterone System Blockade in Diabetic Nephropathy. Present Evidences. J Clin Med. 4(11): 1908-1937. 
McClelland RL, Jorgensen NW, Budoff M, Blaha MJ, Post WS, Kronmal RA, Bild DE, Shea S, Liu K, Watson KE, et al. 2015. 10-Year Coronary Heart Disease Risk Prediction Using Coronary Artery Calcium and Traditional Risk Factors: Derivation in the MESA (Multi-Ethnic Study of Atherosclerosis) With Validation in the HNR (Heinz Nixdorf Recall) Study and the DHS (Dallas Heart Study). J Am Coll Cardiol. 66(15): 1643-1653.

Miller, AJ, Arnold, AC. (2019). The renin-angiotensin system in cardiovascular autonomic control: recent developments and clinical implications. Clin Auton Res. 29(2), 231-243.

Mozaffarian D, Benjamin EJ, Go AS, Arnett DK, Blaha MJ, Cushman M, de Ferranti S, Després, JP, Fullerton HJ, Howard V, et al. American Heart Association Statistics Committee and Stroke Statistics Subcommittee. 2015. Heart disease and stroke statistics--2015 update: a report from the American Heart Association. Circulation. 131(4): e29-e322.

Muñoz M, Rincón J, Pedreañez A, Viera N, Hernández-Fonseca JP, Mosquera J. 2011. Proinflammatory role of angiotensin II in a rat nephrosis model induced by adriamycin. J Renin Angiotensin Aldosterone Syst. 12(4): 404-412.

Ogata Y, Nemoto W, Nakagawasai O, Yamagata R, Tadano T, Tan-No K. 2016. Involvement of Spinal Angiotensin II System in Streptozotocin-Induced Diabetic Neuropathic Pain in Mice. Mol Pharmacol. 90(3): 205-213.

Orea-Tejeda A, Colín-Ramírez E, Castillo-Martínez L, Asensio-Lafuente E, Corzo-León D, GonzálezToledo R, Rebollar-González V, Narváez-David R, \& Dorantes-García J. 2007. Aldosterone receptor antagonists induce favorable cardiac remodeling in diastolic heart failure patients. Rev Invest Clin. 59(2): 103-107.

Pickering RJ, Tikellis C, Rosado CJ, Tsorotes D, Dimitropoulos A, Smith M, Huet O, Seeber R M, Abhayawardana R, Johnstone EK, et al. 2019. Transactivation of RAGE mediates angiotensininduced inflammation and atherogenesis. J Clin Invest. 129(1): 406-421.

Qian X, Lin L, Zong Y, Yuan Y, Dong Y, Fu Y, Shao W, Li Y, Gao Q. 2018. Shifts in renin-angiotensin system components, angiogenesis, and oxidative stress-related protein expression in the lamina cribrosa region of streptozotocin-induced diabetic mice. Graefes Arch Clin Exp Ophthalmol. 256(3): 525-534.

Roscioni SS, Heerspink HJ, de Zeeuw D. 2014. The effect of RAAS blockade on the progression of diabetic nephropathy. Nat Rev Nephrol. 10(2): 77-87.

Scheen AJ. 2004. Renin-angiotensin system inhibition prevents type 2 diabetes mellitus. Part 2. Overview of physiological and biochemical mechanisms. Diabetes Metab. 30(6): 498-505.

Schievink B, Kröpelin T, Mulder S, Parving HH, Remuzzi G, Dwyer J, Vemer P, de Zeeuw D, Lambers Heerspink HJ. 2016. Early renin-angiotensin system intervention is more beneficial than late intervention in delaying end-stage renal disease in patients with type 2 diabetes. Diabetes Obes Metab. 18(1): 64-71.

Singh VP, Le B, Khode R, Baker KM, Kumar R. 2008. Intracellular angiotensin II production in diabetic rats is correlated with cardiomyocyte apoptosis, oxidative stress, and cardiac fibrosis. Diabetes. 57(12): 3297-3306.

Smith DH. 2008. Comparison of angiotensin II type 1 receptor antagonists in the treatment of essential hypertension. Drugs. 68(9): 1207-1225.

Stefano GB, Challenger S, Kream RM. 2016. Hyperglycemia-associated alterations in cellular signaling and dysregulated mitochondrial bioenergetics in human metabolic disorders. Eur J Nutr. 55(8): 2339-2345. 
Teissier T, Boulanger É. 2019. The receptor for advanced glycation end-products (RAGE) is an important pattern recognition receptor (PRR) for inflammaging. Biogerontology. 20(3): 279-301. Urata H, Boehm KD, Philip A, Kinoshita A, Gabrovsek J, Bumpus FM, Husain A. 1993. Cellular localization and regional distribution of an angiotensin II-forming chymase in the heart. J Clin Invest. 91(4): 1269-1281.

Urata H, Kinoshita A, Perez DM, Misono KS, Bumpus FM, Graham RM, Husain A. 1991. Cloning of the gene and cDNA for human heart chymase. J Biol Chem. 266(26): 17173-17179.

Vargas R, Rincón J, Pedreañez A, Viera N, Hernández-Fonseca JP, Peña C, \& Mosquera J. 2012. Role of angiotensin II in the brain inflammatory events during experimental diabetes in rats. Brain Res. 1453: 64-76.

Varma U, Koutsifeli P, Benson VL, Mellor KM, Delbridge L. 2018. Molecular mechanisms of cardiac pathology in diabetes - Experimental insights. Biochim Biophys Acta Mol Basis Dis. 1864(5 Pt B): 1949-1959.

Wang X, Ye Y, Gong H, Wu J, Yuan J, Wang S, Yin P, Ding Z, Kang L, Jiang Q, et al. 2016. The effects of different angiotensin II type 1 receptor blockers on the regulation of the ACE-Angll-AT1 and ACE2-Ang(1-7)-Mas axes in pressure overload-induced cardiac remodeling in male mice. J Mol Cell Cardiol. 97: 180-190.

Westermann D, RutschowS, JägerS, Linderer A, AnkerS, Riad A, UngerT, Schultheiss HP, Pauschinger M, Tschöpe C. 2007. Contributions of inflammation and cardiac matrix metalloproteinase activity to cardiac failure in diabetic cardiomyopathy: the role of angiotensin type 1 receptor antagonism. Diabetes. 56(3): 641-646.

Zhou J, Xu X, Liu JJ, Lin YX, Gao GD. 2007. Angiotensin II receptors subtypes mediate diverse gene expression profile in adult hypertrophic cardiomyocytes. Clin Exp Pharmacol Physiol. 34(11): 1191-1198. 